\section{S125 POTENTIAL INTERACTIONS OF CRACM ION CHANNELS WITH THE CALCIUM ACTIVATED POTASSIUM CHANNEL KCa3.1 IN HUMAN LUNG MAST CELLS}

doi:10.1136/thoraxjnl-2011-201054b.125

${ }^{1}$ I Ashmole, ${ }^{1} \mathrm{M}$ Patel, ${ }^{1} \mathrm{~S} \mathrm{M}$ Duffy, ${ }^{2} \mathrm{M}$ Leyland, ${ }^{1} \mathrm{P}$ Bradding. ${ }^{1}$ Institute for Lung Heath, Dept of Infection, Immunity and Inflammation, University of Leicester, Leicester, UK; ${ }^{2}$ Department of Biochemistry, University of Leicester, Leicester, UK

Introduction and Objectives The influx of extracellular $\mathrm{Ca}^{2+}$ is essential for the IgE-dependent release of both preformed mediators and newly-generated autacoids and cytokines from human lung mast cells (HLMC). Members of the recently discovered CRACM (also known as Orai) ion channel family may be responsible for this influx. These channels carry $\mathrm{Ca}^{2+}$ selective currents $\left(\mathrm{I}_{\mathrm{CRAC}}\right)$ that are activated when endoplasmic reticulum $\mathrm{Ca}^{2+}$ stores are emptied. The major $\mathrm{K}^{+}$selective conductance in HLMC is generated by the $\mathrm{Ca}^{2+}$-activated $\mathrm{K}^{+}$channel $\mathrm{K}_{\mathrm{Ca}} 3$.1. There appears to be a close functional relationship between CRACM and $\mathrm{K}_{\mathrm{Ca}} 3.1$ channels. $\mathrm{K}_{\mathrm{Ca}} 3.1$ channels activated by the influx of extracellular $\mathrm{Ca}^{2+}$ act to hyperpolarise the cell membrane, thus maintaining the driving force on CRACM channels. Here we investigate whether CRACM and $\mathrm{K}_{\mathrm{Ca}} 3.1$ channels physically interact.

Methods Vectors were assembled directing the expression of either CRACM1 or CRACM2 channels tagged at their N-terminus with the c-Myc epitope and human $\mathrm{K}_{\mathrm{Ca}} 3.1$ tagged at its $\mathrm{C}$-terminus with the FLAG epitope. HEK293 cells were transiently transfected to express either a single epitope tagged channel or both a CRACMmyc channel and $\mathrm{K}_{\mathrm{Ca}} 3.1-\mathrm{FLAG}$. Cell lysates were prepared and potential interactions between CRACM and $\mathrm{K}_{\mathrm{Ca}} 3.1$ proteins tested by immunoprecipitation and immunoblotting.

Results Expression of CRACM1-myc, CRACM2-myc and $\mathrm{K}_{\mathrm{Ca}} 3.1$ FLAG proteins was confirmed by immunoblotting of lysates of HEK293 cells transiently transfected with the appropriate vector(s). Using an anti c-myc antibody to immunoprecipitate CRACM1-myc protein, $\mathrm{K}_{\mathrm{Ca}}$ 3.1-FLAG was found to be co-immunoprecipitated. Coimmunoprecipitation of $\mathrm{K}_{\mathrm{Ca}} 3.1-\mathrm{FLAG}$ was observed only when it was co-expressed with CRACM1-myc. Similarly, using an antiFLAG antibody to immunoprecipitate $\mathrm{K}_{\mathrm{Ca}} 3.1$-FLAG protein, CRACM1-myc was co-immunoprecipitated. Again co-immunoprecipitation was dependent on both the CRACM1-myc and $\mathrm{K}_{\mathrm{Ca}} 3.1$ FLAG proteins being expressed together. In contrast, under identical reaction conditions, no co-immunoprecipitation of $\mathrm{K}_{\mathrm{Ca}} 3$.1-FLAG was observed when co-expressed with CRACM2-myc.

Conclusions The co-immunoprecipitation of $\mathrm{K}_{\mathrm{Ca}} 3.1$-FLAG with CRACM1-myc and vice versa provides evidence that these channels do physically interact. Such an interaction in HLMC would make both channels a potential therapeutic target in the treatment of asthma and would allow $\mathrm{K}_{\mathrm{Ca}} 3.1$ to be added to the growing list of CRACM1 protein binding partners.

\section{S126 THE CELL ADHESION RECEPTOR CADM1 REGULATES OTHER ADHESION RECEPTORS IN HUMAN MAST CELLS}

doi:10.1136/thoraxjnl-2011-201054b.126

E P Moiseeva, M L Leyland, P Bradding. University of Leicester, Leicester, UK

Introduction and Objectives Cell adhesion molecule 1 (CADM1) contributes to adhesion, viability and adhesion-induced degranulation in mast cells. Severity of asthma correlates with accumulation of human lung mast cells in specific compartments, including airway smooth muscle (ASM). human lung mast cells adhesion to ASM is known to be mediated by CADM1, integrins and other receptors. Here we investigated the role of CADM1 in the cell-cell and extracellular matrix (ECM) adhesion of mast cells to primary ASM.
Methods The mast cell line HMC-1 was used in adhesion assays to primary ASM and ECM produced by these cells. ECM was prepared by hypotonic extraction with detergents to remove cell content. Modulation of CADM1 expression by adenoviral delivery in HMC-1 cells was verified by FACS and Western blotting. Cells with modulated CADM1 were examined in adhesion assays.

Results HMC- 1 cells adhered to ASM in a time-dependent manner, reaching $92 \%$ and $70 \%$ adhesion at $1 \mathrm{~h}$ to ASM and ECM, respectively. Addition of EDTA resulted in a reduction of adhesion to ASM and ECM to $55 \%$ and $22 \%$, respectively, indicating a major role of cation $^{2+}$-independent receptors in cell-cell and integrins in ECM adhesion of mast cells. CADM1-overexpression did not change adhesion to ASM (98\%), but decreased adhesion to ECM to $55 \%$. CADM1 RNA interference in HMC-1 resulted in $60 \%$ reduction of surface CADM1 and complete loss of CADM1 determined by FACS and Western blotting, respectively, 6 days after transduction. CADM1 downregulation resulted in drastically reduced adhesion to both ASM and ECM down to 37 and $17 \%$, respectively. Addition of EDTA further decreased adhesion to ASM and ECM to $13 \%$ and $6 \%$, respectively. Thus, CADM1 downregulation drastically decreased net adhesion of mast cells.

Conclusions Mast cell adhesion receptor CADM1 plays a regulatory role in mast cell adhesion by affecting not only cell-cell adhesion, but also ECM adhesion.

\section{Lung infections: mechanisms of disease S127 ANTI-PROTEIN SEROLOGICAL RESPONSES TO STREPTOCOCCUS PNEUMONIAE, IN DIVERSE POPULATIONS}

doi:10.1136/thoraxjnl-2011-201054b.127

${ }^{1} \mathrm{R}$ J Wilson, ${ }^{1} \mathrm{~J} \mathrm{M}$ Cohen, ${ }^{1} \mathrm{C}$ Hyams, ${ }^{2} \mathrm{~W} \mathrm{~J}$ van Wamel, ${ }^{2} \mathrm{C}$ de Vogel, ${ }^{2} \mathrm{~A}$ van Belkum, ${ }^{3} \mathrm{~S}$ B Gordon, ' IJ S Brown. 'University College London, London, UK; ' ${ }^{2}$ Erasmus MC, Rotterdam, Netherlands; ${ }^{3}$ Liverpool School of Tropical Medicine, Liverpool, UK

Although vaccine-induced immunity to Streptococcus pneumoniae depends on anti-capsule antibody responses, naturally acquired adaptive immunity may also depend on antibody against surface protein antigens. Unlike capsular antigen, protein antigens are often highly conserved between $S$ pneumoniae strains and thus could be effective vaccines against all $S$ pneumoniae capsular serotypes. We have investigated the pattern of naturally acquired antibodies to $S$ pneumoniae protein antigens in diverse populations and assessed whether they could be protective. Immunoblots against lysates of wild-type and mutant $S$ pneumoniae with sera from adult humans demonstrated antibody responses to a large number of protein antigens including PspC, PspA and PhtD. Using a multiplex assay of 18 recombinant pneumococcal antigens conjugated to fluorescent beads (xMAP) we made semi-quantitative assessments of serological responses in sera obtained from individuals from the UK and Malawi, as well as in commercial immunoglobulin (IVIG) preparations, pooled from donors in either Europe or USA. All individuals, in both geographical populations had significant levels of antibody to a number of pneumococcal proteins. Individuals varied in their response to specific pneumococcal antigens, with absent responses to some antigens that were dominant in other subjects. However, pooled sera from Malawi and IVIG products from both Europe and the USA had remarkably similar patterns of antigen dominance, with consistently high levels of antibody responses to the antigens $\mathrm{PhtD}, \mathrm{PspC}$, PspA and PsaA and weak responses to PilusA, Eno, NanA and SlrA. To investigate the functional importance of these protein antigens we used in vitro flow cytometry assays of complement deposition and neutrophil phagocytosis using bacteria 